\title{
A NECESSARY AND SUFFICIENT CONDITION FOR THE ASYMPTOTIC VERSION OF AHLFORS' DISTORTION PROPERTY ${ }^{1}$
}

BY

BURTON RODIN AND S. E. WARSCHAWSKI

\begin{abstract}
Let $f$ be a conformal map of $R=\left\{w=u+i v \in \mathbf{C} \mid \varphi_{0}(u)<v<\varphi_{1}(u)\right\}$ onto $S=\{z=x+i y \in \mathbf{C} \mid 0<y<1\}$ where the $\varphi_{j} \in C^{0}(-\infty, \infty)$ and $\operatorname{Re} f(w) \rightarrow$ $\pm \infty$ as $\operatorname{Re} w \rightarrow \pm \infty$. There are well-known results giving conditions on $R$ sufficient for the distortion property $\operatorname{Re} f(u+i v)=\int_{0}^{u}\left(\varphi_{1}-\varphi_{0}\right)^{-1} d u+$ const. $+o(1)$, where $o(1) \rightarrow 0$ as $u \rightarrow+\infty$. In this paper the authors give a condition on $R$ which is both necessary and sufficient for $f$ to have this property.
\end{abstract}

Let $R \subset \mathrm{C}$ be a region of the form $R=\left\{w=u+i v \mid \varphi_{0}(u)<v<\varphi_{1}(u)\right\}$ where $\varphi_{0}$ and $\varphi_{1}$ are extended real valued functions defined for $-\infty<u<+\infty$. For the sake of simplicity we shall require $\varphi_{0}$ and $\varphi_{1}$ to be continuous. Let $\theta(u)=\varphi_{1}(u)-$ $\varphi_{0}(u)$.

Let $S \subset \mathrm{C}$ be the parallel strip $S=\{z=x+i y \mid 0<y<1\}$. Let $w=F(z)$ be a one-to-one conformal map of $S$ onto $R$ such that $\operatorname{Re} F(z) \rightarrow \pm \infty$ as $\operatorname{Re} z \rightarrow \pm \infty$, respectively. Let $z=f(w)$ be the inverse mapping.

Inequalities of the form

$$
m \leqslant \operatorname{Re} f(w)-\int_{0}^{u} \frac{d u}{\theta(u)} \leqslant M \quad(w=u+i v)
$$

were first investigated in Ahlfors [1] (the left hand inequality corresponds to his Distortion Theorem; the right hand inequality to his Second Fundamental Inequality). That work stimulated efforts to find other properties of $R$ which imply the validity of (a).

The problem takes a simpler form when Eke's theorem [2, Theorem 2] is applied (cf. also [8, Theorem 3]). This theorem shows that (a) holds for $0<u<\infty$ if and only if the center term actually tends to a limit

$$
\operatorname{Re} f(w)=\int_{0}^{u} \frac{d u}{\theta(u)}+\text { const. }+o(1) \quad(w=u+i v),
$$

where $o(1) \rightarrow 0$ as $\operatorname{Re} w \rightarrow+\infty$.

Ahlfors' original results, as strengthened by Jenkins-Oikawa [4], show that (A) holds if $R$ contains the real axis and its boundary curves $\varphi_{0}, \varphi_{1}$ are of bounded

Received by the editors December 1, 1981 and, in revised form, March 2, 1982.

1980 Mathematics Subject Classification. Primary 30A30.

'Research supported in part by the National Science Foundation. 
variation and bounded away from zero. A number of other geometric properties of $R$ which imply (A) have been discovered. Examples of such sufficient conditions are the bounded 2/3-variation condition of Jenkins-Oikawa [5], the convergence of the integral $\int_{0}^{\infty} \varphi_{j}^{\prime 2}\left(1+\left|\varphi_{j}^{\prime}\right|\right)^{-1} d u$ for $j=0,1$ (Lelong-Ferrand [3], Rodin-Warschawski [10]), and the convergence of $\int_{0}^{\infty}\left(\varphi_{0}^{\prime 2}+\varphi_{1}^{\prime 2}\right) \theta^{-1} d u$ (Warschawski [11], RodinWarschawski [8]). None of these sufficient conditions is also necessary.

In Theorem 1 below we give a complete solution to the problem of finding geometric conditions on $R$ which are both necessary and sufficient for property (A).

REMARK. The sufficient condition of [11] referred to above can be derived directly from Theorem 1 by taking $\alpha(u, t)=t \varphi_{1}(u)+(1-t) \varphi_{0}(u)$. It is not evident if the other sufficient conditions can be derived from Theorem 1 in a direct manner.

1. A class $C^{1}$ real valued function $\alpha(u, t)$ defined for $-\infty<u<+\infty, 0<t<1$ will be called a stratification of $R$ if $u+i \alpha(u, t) \in R$ for all $(u, t)$ in the domain of $\alpha$. For simplicity we shall also require $\alpha_{t}>0$.

THEOREM 1. A necessary and sufficient condition for $R$ to have property (A) is that $R$ admit a stratification $\alpha(u, t)$ such that the integrals

$$
\int_{0}^{1} \int_{u^{\prime}}^{u^{\prime \prime}}\left(\frac{1}{\theta(u)}-\frac{1}{\alpha_{t}(u, t)}\right) d u d t
$$

and

$$
\int_{0}^{1} \int_{u^{\prime}}^{u^{\prime \prime}} \frac{\alpha_{u}^{2}(u, t)}{\alpha_{t}(u, t)} d u d t
$$

remain bounded above and below as $u^{\prime}, u^{\prime \prime} \rightarrow+\infty$.

Proof. Necessity. Since the angle of inclination of any chord of a boundary component of $R$ is less than $\pi / 2$ in magnitude, it follows that $\left|\operatorname{Arg} F^{\prime}(z)\right|<\pi / 2$; a detailed proof is given in Lemma 2 of $\$ 2$. Hence for each fixed $t \in(0,1)$ the stream line $\{w \in R \mid \operatorname{Im} f(w)=t\}$ is the graph of a function. Denote this function by $u \mapsto \alpha(u, t)$. We shall show that this $\alpha$ is a stratification of $R$ which satisfies the boundedness conditions of the theorem.

By the definition of $\alpha$ we have

$$
v(x, t)=\alpha(u(x, t), t)
$$

where $F(x+i t)=u(x, t)+i v(x, t)$. Take partial derivatives with respect to $x, t$ in (3) to obtain

$$
\alpha_{u}(u, t)=-\frac{\operatorname{Im} f^{\prime}(w)}{\operatorname{Re} f^{\prime}(w)}, \quad \alpha_{t}(u, t)=\frac{1}{\operatorname{Re} f^{\prime}(w)} \quad(w=u+i \alpha(u, t)) .
$$


The integral (2) for this $\alpha$ can be estimated as follows, where $u^{\prime}<u^{\prime \prime}$ :

$$
\begin{aligned}
\int_{0}^{1} \int_{u^{\prime}}^{u^{\prime \prime}} \frac{\alpha_{u}^{2}}{\alpha_{t}} d u d t & =\int_{0}^{1} \int_{u^{\prime}}^{u^{\prime \prime}} \frac{\operatorname{Im}^{2} f^{\prime}}{\operatorname{Re} f^{\prime}} d u d t \\
& =\int_{0}^{1} \int_{x\left(u^{\prime}, \alpha\left(u^{\prime}, t\right)\right)}^{x\left(u^{\prime \prime}, \alpha\left(u^{\prime \prime}, t\right)\right)} \frac{\operatorname{Im}^{2} f^{\prime}}{\operatorname{Re} f^{\prime}}\left(\cos \operatorname{Arg} F^{\prime}\right)\left|F^{\prime}\right| d x d t \\
& =\int_{0}^{1} \int_{x\left(u^{\prime}, \alpha\left(u^{\prime}, t\right)\right)}^{x\left(u^{\prime \prime}, \alpha\left(u^{\prime \prime}, t\right)\right)} \sin ^{2} \operatorname{Arg} F^{\prime} d x d t \\
& \leqslant \int_{0}^{1} \int_{x_{0}}^{+\infty} \operatorname{Arg}^{2} F^{\prime} d x d t
\end{aligned}
$$

for suitable $x_{0}$. By Theorem 5 of Rodin-Warschawski [9] (see Lemma 1 in $\$ 2$ for a selfcontained proof of this fact in the present, less general, context) the last integral is finite under our hypothesis that $R$ satisfies property (A).

The integral (1) for this $\alpha$ can be transformed as follows:

$$
\begin{aligned}
\int_{0}^{1} \int_{u^{\prime}}^{u^{\prime \prime}}\left(\frac{1}{\theta(u)}-\frac{1}{\alpha_{t}(u, t)}\right) d u d t=\int_{0}^{1} \int_{u^{\prime}}^{u^{\prime \prime}}\left(\frac{1}{\theta(u)}-\operatorname{Re} f^{\prime}\right) d u d t \\
=\int_{u^{\prime}}^{u^{\prime \prime}} \frac{d u}{\theta(u)}-\int_{0}^{1} \int_{x\left(u^{\prime}, \alpha\left(u^{\prime}, t\right)\right)}^{x\left(u^{\prime \prime}, \alpha\left(u^{\prime \prime}, t\right)\right)} \operatorname{Re} f^{\prime} \cdot \cos \operatorname{Arg} f^{\prime} \cdot\left|F^{\prime}\right| d x d t \\
=\int_{u^{\prime}}^{u^{\prime \prime}} \frac{d u}{\theta(u)}-\int_{0}^{1} \int_{x^{\prime}}^{x^{\prime \prime}} \cos ^{2} \operatorname{Arg} F^{\prime} d x d t \\
=\int_{u^{\prime}}^{u^{\prime \prime}} \frac{d u}{\theta(u)}-\int_{0}^{1} \int_{x^{\prime}}^{x^{\prime \prime}} d x d y+\int_{0}^{1} \int_{x^{\prime}}^{x^{\prime \prime}} \sin ^{2} \operatorname{Arg} F^{\prime} d x d t
\end{aligned}
$$

where $x^{\prime}=x\left(u^{\prime}, \alpha\left(u^{\prime}, t\right)\right)$ and $x^{\prime \prime}=x\left(u^{\prime \prime}, \alpha\left(u^{\prime \prime}, t\right)\right)$. As already noted, this last integral is bounded under the assumption that property (A) holds. The remaining term

$$
\int_{u^{\prime}}^{u^{\prime \prime}} \frac{d u}{\theta(u)}-\int_{0}^{1} \int_{x^{\prime}}^{x^{\prime \prime}} d x d y=\int_{u^{\prime}}^{u^{\prime \prime}} \frac{d u}{\theta(u)}-\iint_{f\left(R\left(u^{\prime}, u^{\prime \prime}\right)\right)} d x d y
$$

where $R\left(u^{\prime}, u^{\prime \prime}\right)=\left\{w \in R \mid u^{\prime}<\operatorname{Re} w<u^{\prime \prime}\right\}$, is also bounded. Indeed, note that property (A) implies that the horizontal oscillation

$$
\omega(u)=\sup \left\{\operatorname{Re} f\left(w_{2}\right)-\operatorname{Re} f\left(w_{1}\right) \mid w_{1}, w_{2} \in R \text { and } \operatorname{Re} w_{1}=\operatorname{Re} w_{2}=u\right\}
$$

tends to zero as $u \rightarrow+\infty$. Let $x^{\prime \prime}-x^{\prime}=\operatorname{Re} f\left(u^{\prime \prime}+i v^{\prime \prime}\right)-\operatorname{Re} f\left(u^{\prime}+i v^{\prime}\right)$ where $u^{\prime}+i v^{\prime} \in R, u^{\prime \prime}+i v^{\prime \prime} \in R$, and $u^{\prime}<u^{\prime \prime}$. Then the assertion of boundedness follows from

$$
x^{\prime \prime}-x^{\prime}-\omega\left(u^{\prime}\right)-\omega\left(u^{\prime \prime}\right) \leqslant \iint_{f\left(R\left(u^{\prime}, u^{\prime \prime}\right)\right)} d x d y \leqslant x^{\prime \prime}-x^{\prime}+\omega\left(u^{\prime}\right)+\omega\left(u^{\prime \prime}\right),
$$

the consequence of property (A),

$$
x^{\prime \prime}-x^{\prime}=\int_{u^{\prime}}^{u^{\prime \prime}} \frac{d u}{\theta(u)}+O(1)
$$

and the fact $\omega(u)=o(1)$ mentioned above. 
Sufficiency. We now assume that $\alpha(u, t)$ is a stratification of $R$ such that the integrals (1) and (2) are bounded. For given $0 \leqslant u^{\prime}<u^{\prime \prime}$ consider the curve family $\left\{\gamma_{t}\right\}_{0<t<1}$ defined by $u \mapsto \gamma_{t}(u)=u+i \alpha(u, t)$ for $u^{\prime} \leqslant u \leqslant u^{\prime \prime}$. By well-known properties of extremal length we have

$$
\int_{u^{\prime}}^{u^{\prime \prime}} \frac{d u}{\theta(u)} \leqslant \lambda_{R}\left(u^{\prime}, u^{\prime \prime}\right) \leqslant \lambda\left(\left\{\gamma_{t}\right\}_{0<t<1}\right)
$$

where $\lambda_{R}\left(u^{\prime}, u^{\prime \prime}\right)$ is the extremal distance between the vertical sides in $\left\{w \in R \mid u^{\prime}<\right.$ $\left.\operatorname{Re} w<u^{\prime \prime}\right\}$. We shall show that $\lambda\left(\left\{\gamma_{t}\right\}_{0<t<1}\right) \leqslant \int_{u^{\prime}}^{u^{\prime \prime}} \theta^{-1}(u) d u+O(1)$ where $O(1)$ is bounded for all $0 \leqslant u^{\prime}<u^{\prime \prime}$. It will then follow that property (A) holds (see Theorem 3 of Rodin-Warschawski [8]; cf. also Eke [2, Theorem 2]).

Since $\left\{\gamma_{t}\right\}_{0<t<1}$ is a 1-parameter curve family one can calculate its extremal length exactly (see, for example, Theorem 14 of [6]). Define a map of $\{0<u<+\infty, 0<t$ $<1$ ) into $R$, denoted $u+i t \mapsto c(u, t)=u+i v$, by letting $v=\alpha(u, t)$. Then

$$
\begin{gathered}
J(u, t) \equiv \frac{\partial(u, v)}{\partial(u, t)}=\left|\begin{array}{cc}
1 & 0 \\
\alpha_{u} & \alpha_{t}
\end{array}\right|=\alpha_{t}, \\
\left|\frac{\partial c(u, t)}{\partial u}\right|^{2}=1+\alpha_{u}^{2}, \\
l(t) \equiv \int_{u^{\prime}}^{u^{\prime \prime}} \frac{|\partial c / \partial u|^{2}}{J} d u=\int_{u^{\prime}}^{u^{\prime \prime}} \frac{1+\alpha_{u}^{2}}{\alpha_{t}} d u .
\end{gathered}
$$

One has

$$
\begin{aligned}
\lambda\left(\left\{\gamma_{t}\right\}\right) & =\left(\int_{0}^{1} \frac{d t}{l(t)}\right)^{-1} \leqslant \int_{0}^{1} l(t) d t=\int_{0}^{1} \int_{u^{\prime}}^{u^{\prime \prime}}\left(\frac{1+\alpha_{u}^{2}}{\alpha_{t}}\right) d u d t \\
& =\int_{0}^{1} \int_{u^{\prime}}^{u^{\prime \prime}} \frac{1}{\alpha_{t}} d u d t+\int_{0}^{1} \int_{u^{\prime}}^{u^{\prime \prime}} \frac{\alpha_{u}^{2}}{\alpha_{t}} d u d t
\end{aligned}
$$

Our hypothesis on the boundedness of integrals (1) and (2) means that the sum of

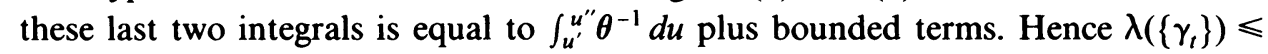
$\int_{u^{\prime}}^{u^{\prime \prime}} \theta^{-1} d u+O(1)$ as desired.

2. We now prove the two lemmas referred to in the necessity part of the proof of Theorem 1.

LEMMA 1. Suppose $f: R \rightarrow S$ satisifies property (A). Then the inverse function $F$ : $S \rightarrow R$ satisfies

$$
\iint_{\substack{0<x<\infty \\ 0<y<1}} \operatorname{Arg}^{2} F^{\prime}(z) d x d y<\infty
$$


Proof. Let $R(a, b)=R \cap\{w \mid a<\operatorname{Re} w<b\}$. Let $l(u)$ be the length of $f\left(\theta_{u}\right)$, where $\theta_{u}=\left\{w \mid \operatorname{Re} w=u, \varphi_{0}(u)<\operatorname{Im} w<\varphi_{1}(u)\right\}$. We have

$$
\begin{aligned}
0 & \leqslant \iint_{R(0, u)}\left[\frac{1}{\theta(u)}-\left|f^{\prime}(w)\right|\right]^{2} d u d v \\
& =\int_{0}^{u} \frac{d u}{\theta(u)}-2 \int_{0}^{u} \frac{l(u)}{\theta(u)} d u+\iint_{R(0, u)}\left|f^{\prime}(w)\right|^{2} d u d v \\
& =\left\{\iint_{R(0, u)}\left|f^{\prime}(w)\right|^{2} d u d v-\int_{0}^{u} \frac{d u}{\theta(u)}\right\}-2 \int_{0}^{u} \frac{l(u)-1}{\theta(u)} d u .
\end{aligned}
$$

We have already seen that the term in braces is uniformly bounded for $0<u<\infty$ (see the last paragraph of the Necessity part of the proof of Theorem 1). The last integral above is nonnegative since $l(u) \geqslant 1$. Hence

$$
\begin{gathered}
\iint_{R(0, u)}\left[\frac{1}{\theta(u)}-\left|f^{\prime}(w)\right|\right]^{2} d u d v=O(1), \\
\int_{0}^{u} \frac{l(u)-1}{\theta(u)} d u=O(1) .
\end{gathered}
$$

(REMARK. With more work one can show that $\int_{0}^{u}\left(l^{2}(u)-1\right) / \theta(u) d u=O(1)$; see Theorem 1 of [9].)

For $0<t<1$ let $\gamma_{t}$ be the part of the stream line $\{w \mid \operatorname{Im} f(w)=t\}$ which lies in $R(0, u) . \gamma_{t}$ is a connected set since $\left|\operatorname{Arg} F^{\prime}(z)\right|<\pi / 2$. We have

$$
\int_{0}^{u} \frac{d u}{\theta(u)} \leqslant \int_{\gamma_{t}} \frac{|d w|}{\theta(u)}=\int_{f\left(\gamma_{t}\right)} \frac{\left|F^{\prime}(z)\right| d x}{\theta(u(z))} .
$$

After integrating for $t \in(0,1)$ we obtain

$$
\int_{0}^{u} \frac{d u}{\theta} \leqslant \int_{0}^{1} \int_{\gamma_{t}} \frac{|d w|}{\theta} d t=\iint_{f(R(0, u))} \frac{\left|F^{\prime}\right|}{\theta} d x d y=\iint_{R(0, u)} \frac{\left|f^{\prime}\right|}{\theta} d u d v .
$$

The last integral can be rewritten as $\int_{0}^{u} l(u) \theta^{-1}(u) d u$ which, in view of (6), is equal to $\int_{0}^{u} \theta^{-1}(u) d u+O(1)$. We conclude that

$$
\int_{0}^{1} \int_{\gamma_{t}} \frac{|d w|-d u}{\theta(u)} d t=O(1)
$$

Replace $d u$ by $|d w| \cos \operatorname{Arg} f^{\prime}(w)$ and transform the above integral to

$$
\int_{0}^{1} \int_{\gamma_{t}} \frac{1-\cos \operatorname{Arg} f^{\prime}(w)}{\theta}|d w| d t=\iint_{f(R(0, u))}\left(1-\cos \operatorname{Arg} f^{\prime}(w(z))\right) \frac{\left|F^{\prime}\right|}{\theta} d x d y .
$$

Thus

$$
\iint_{f(R(0, u))}\left(1-\cos \operatorname{Arg} F^{\prime}(z)\right) \frac{\left|F^{\prime}\right|}{\theta} d x d y=O(1)
$$


A change of variables in (5) leads to

$$
\iint_{f(R(0, u))}\left(\frac{F^{\prime}}{\theta}-1\right)^{2} d x d y=O(1) .
$$

It follows from (7) and (8) that

$$
\iint_{f(R(0, u))}\left(1-\cos \operatorname{Arg} F^{\prime}(w)\right) d x d y=O(1) ;
$$

indeed, (8) shows that the set $E_{1}=\left\{z|| F^{\prime}(z) \mid / \theta(u(z)) \leqslant \frac{1}{2}\right\}$ has finite area and hence

$$
\iint_{E_{1}}\left(1-\cos \operatorname{Arg} F^{\prime}(z)\right) d x d y<\infty .
$$

On the complementary set $E_{2}=\left\{z|| F^{\prime}(z) \mid / \theta(u(z))>\frac{1}{2}\right\}$ equation (7) shows that

$$
\iint_{E_{2}}\left(1-\cos \operatorname{Arg} F^{\prime}(z)\right) d x d y<\infty .
$$

Therefore

$$
\iint_{\substack{0<x<\infty \\ 0<y<1}}\left(1-\cos \operatorname{Arg} F^{\prime}(z)\right) d x d y<\infty .
$$

The estimate $1-\cos \beta \geqslant\left(4 / \pi^{2}\right) \beta^{2}$ is valid in the range $|\beta| \leqslant \pi / 2$. When this is applied to (10) we obtain $\iint \operatorname{Arg}^{2} F^{\prime}(z) d x d y<\infty$ as asserted. This completes the proof of Lemma 1.

LeMma 2. The map $F: S \rightarrow R$ satisfies $\left|\operatorname{Arg} F^{\prime}(z)\right|<\pi / 2$ for all $z \in S$.

Proof. The proof is modeled in part after the argument in [7, pp. 102-104]. Let $R_{a}=R \cap\{w=u+i v \mid \operatorname{Re} u>a\}$ for some fixed $a$ and let $G$ map the half-strip $S_{1}=\{0<x<\infty, 0<y<1\}$ conformally and one-to-one onto $R_{a}$ such that 0 and $i$ correspond to $w=a+i \varphi_{0}(a)$ and $w=a+i \varphi_{1}(a)$, respectively, and

$$
\lim _{x \rightarrow+\infty} \operatorname{Re} G(z)=+\infty \text {. }
$$

We show first that

$$
\left|\operatorname{Arg} G^{\prime}(z)\right| \leqslant \frac{\pi}{2} \quad \text { for } z \in S_{1} .
$$

For $b>a$ we consider the quadrilateral

$$
Q=\left\{a<u<b, \varphi_{0}(u)<v<\varphi_{1}(u)\right\} .
$$

Then there exists a unique $\beta>0$ and a one-to-one conformal map $g$ of the rectangle $T=\{0<x<\beta, 0<y<1\}$ onto $Q$ such that the vertices $0, \beta, i \beta$ and $i$ of $T$ correspond to the vertices $a+i \varphi_{0}(a), b+i \varphi_{0}(b), b+i \varphi_{1}(b), a+i \varphi_{1}(a)$, respectively. We reflect $T$ in the line $x=\beta$ and obtain a symmetrical rectangle $T^{\prime}$ and an analytic extension of $g$ which maps $T^{\prime}$ onto a quadrangle $Q^{\prime}$ symmetrical to $Q$ with respect to the line $u=b$. For fixed $h>0(h<\beta)$ we define now

$$
P(z, h)=P(z, h ; g)=\arg \frac{g(z+h)-g(z)}{h},
$$


where the branch of the argument is determined to coincide with the principal branch at $z=0$. The geometry of the situation shows that $|P(0, h)|<\pi / 2$ and that $P(z, h)$ extends continuously to $\mathrm{Cl} T$. As $z$ describes the boundary of $T,|P(z, h)|$ remains bounded by $\pi / 2$. Since $P$ is harmonic in $T$ and continuous in $\mathrm{Cl} T$, $|P(z, h)|<\pi / 2$ for all $z \in T$. Thus the continuous argument function in (12) is actually the principal branch everywhere.

We choose now a sequence $\left\{b_{n}\right\}$ with $b_{n} \nearrow+\infty$ as $n \rightarrow \infty$ and determine a corresponding sequence $\left\{\beta_{n}\right\}$ such that the rectangle $T_{n}=\left\{0<x<\beta_{n}, 0<y<1\right\}$ is mapped conformally onto the quadrilateral $Q_{n}=\left\{a<u<b_{n}, \varphi_{0}(u)<v<\varphi_{1}(u)\right\}$ with vertices of $T_{n}$ corresponding to those of $Q_{n}$ as indicated above. If $g_{n}$ denotes the mapping function, it follows as in [7, p. 303] that $\lim _{n \rightarrow \infty} g_{n}(z)=G(z)$, uniformly in any compact subset of $S_{1}$. Hence, uniformly in any compact subset of $S_{1}$

$$
P\left(z, h ; g_{n}\right) \rightarrow P(z, h ; G)=\operatorname{Arg} \frac{G(z+h)-G(z)}{h} \quad \text { as } n \rightarrow \infty,
$$

and then

$$
|P(z, h ; G)| \leqslant \pi / 2 \text { for } z \in S_{1} .
$$

Letting $h \rightarrow 0$ we obtain (11).

Next we observe that $f$, the inverse of $F$, maps $R_{a}$ onto a subregion $f\left(R_{a}\right) \subset S$ (pictured in the $\zeta=\xi+i \eta$ plane). If $\theta_{a}$ denotes a crosscut $\left\{u=a, \varphi_{0}(a)<v<\right.$ $\left.\varphi_{1}(a)\right\}$ of $R$ which determines $R_{a}$, then $f\left(R_{a}\right)$ is bounded by the $\operatorname{arc} \gamma=f\left(\theta_{a}\right)$ and the two half-lines on $\eta=0$ and $\eta=1$ which extend from the endpoints of $\gamma$ to $+\infty$. Let $\psi: S_{1} \rightarrow f\left(R_{a}\right)$ be the one-to-one conformal map of $S_{1}$ onto $f\left(R_{a}\right)$ such that $z=0$ and $z=i$ correspond to the endpoints of $\gamma$ and $\lim _{x \rightarrow+\infty} \operatorname{Re} \psi(z)=+\infty$. Then $G(z)=F(\psi(z))$ and thus $G^{\prime}(z)=F^{\prime}(\psi(z)) \cdot \psi^{\prime}(z)$. It is an elementary fact that $\lim _{k \rightarrow \infty} \psi^{\prime}(z)$ exists for unrestricted approach in $S_{1}$ and is positive. Hence given any $\varepsilon>0$ there exists an $x_{0}=x_{0}(\varepsilon)$ such that (by (11))

$$
\left|\operatorname{Arg} F^{\prime}(\psi(z))\right| \leqslant\left|\operatorname{Arg} G^{\prime}(z)\right|+\varepsilon \leqslant \pi / 2+\varepsilon
$$

for $\operatorname{Re} z \geqslant x_{0}, 0<y<1$.

Returning to $f\left(R_{a}\right)$ in the $\zeta$-plane we can, given $\varepsilon$, determine a $\xi_{0}=\xi_{0}(z)$ such that

$$
\left|\operatorname{Arg} F^{\prime}(\zeta)\right| \leqslant \pi / 2+\varepsilon \text { for } \xi \geqslant \xi_{0}(\varepsilon) \text { and } 0<\eta<1 .
$$

In an analogous manner-by choosing $R_{a}$ as the subregion of $R$ determined by $\theta_{a}$ to the left of $\theta_{a}$-we can establish that for every $\varepsilon>0$ there exists a $\xi_{1}=\xi_{1}(\varepsilon)$ such that

$$
\left|\operatorname{Arg} F^{\prime}(\zeta)\right| \leqslant \pi / 2+\varepsilon \text { for } \xi \leqslant \xi_{1}(\varepsilon) \text { and } 0<\eta<1 .
$$

To complete the proof we consider the rectangle

$$
\left\{\zeta=\xi+i \eta \mid \xi_{1}(\varepsilon) \leqslant \xi \leqslant \xi_{0}(\varepsilon), 0 \leqslant y \leqslant 1\right\}
$$

for fixed $h>0$

$$
P(\zeta, h ; F)=\arg \frac{F(\zeta+h)-F(\zeta)}{h}
$$


Again, we see from the geometry that for $\zeta$ on the horizontal sides of (15) we have by choosing the principal view

$$
|P(\zeta, h ; F)|<\pi / 2 \text {. }
$$

We can continue $P(\zeta)$ as a harmonic function into $S$. Since for $\zeta \in S$

$$
\arg \frac{F(\zeta+h)-F(\zeta)}{h}=\arg F^{\prime}(\zeta+\alpha h), \quad 0<\alpha<1,
$$

where the same determination of the argument is taken on both sides, we see from (13) and (14) that the continuation of $P$ along the two vertical sides of (15) remain the principal value and that

$$
|P(\zeta, h ; F)| \leqslant \pi / 2+\varepsilon .
$$

Hence we have on the boundary and therefore in the interior of the rectangle

$$
\left|\operatorname{Arg} \frac{F(\zeta+h)-F(\zeta)}{h}\right| \leqslant \pi / 2+\varepsilon .
$$

Letting $h \rightarrow 0$ we obtain $\left|\operatorname{Arg} F^{\prime}(\zeta)\right| \leqslant \pi / 2+\varepsilon$ for $\zeta$ in (15). Since $\varepsilon$ is arbitrary we obtain $\left|\operatorname{Arg} F^{\prime}(s)\right| \leqslant \pi / 2$ for $\zeta \in S$. By the maximum principal the strict inequality holds.

\section{REFERENCES}

1. L. V. Ahlfors, Untersuchungen zur Theorie der konformen-Abbildungen und der ganzen Funktionen, Ann. Acad. Sci. Fenn. Ser. AI Math. 9 (1930), 1-40.

2. B. G. Eke, Remarks on Ahlfors' distortion theorem, J. Analyse Math. 19 (1967), 97-134.

3. J. Lelong-Ferrand, Représentation conforme et transformations à intégrale de Dirichlet bornée, Gauthier-Villars, Paris, 1955.

4. J. A. Jenkins and K. Oikawa, On results of Ahlfors and Hayman, Illinois J. Math. 15 (1971), $664-671$.

5. __ On Ahlfors' "second fundamental inequality”, Proc. Amer. Math. Soc. 62 (1977), 266-270.

6. B. Rodin, The method of extremal length, Bull. Amer. Math. Soc. 80 (1974), 587-606.

7. B. Rodin and S. E. Warschawski, On conformal mapping of L-strips, J. London Math. Soc. (2) 11 (1975), 301-307.

8. Extremal length and the boundary behavior of conformal mappings, Ann. Acad. Sci. Fenn. Ser. AI Math. 2 (1976), 467-500.

9. Extremal length and univalent functions III. Consequences of the Ahlfors distortion property, Bull. Inst. Math. Acad. Sinica 6 (1978), 583-597.

10. Extremal length and univalent functions II. Integral estimates of strip mappings, J. Math. Soc. Japan 31 (1979), 87-99.

11. S. E. Warschawski, On conformal mapping of infinite strips, Trans. Amer. Math. Soc. 51 (1942), 280-335.

Department of Mathematics, University of California-San Diego, la Jolla, California 92093 